\title{
EVALUATION OF TRIDIMENTIONAL COMPUTED TOMOGRAPHY IN ASSESSMENT OF MANDIBULAR FRACTURE
}

\author{
Iman Dakhli*
}

\begin{abstract}
Aim: To demonstrate the validity of conventional CT and reconstructed 3DCT in assessment of mandibular fractures. A total of 14 mandibular fractures sustained by 10 patients were evaluated in the current work. All cases were males with ages ranging between 30-50 years. Patients were all assessed by 2DCT and reconstructed CT after compiling detailed history as well as thorough physical examination. The present study strengthens the role of the 3D-CT technique as a supplementary technique to the 2D-CT in the assessment of the mandibular fractures. 3DCT imaging has proved its validity as an exquisite tool in providing higher sensitivity in anatomic localization of mandibular fractures, in detecting comminution and bone displacement.
\end{abstract}

\section{INTRODUCTION}

Maxillofacial fractures occur when the facial bones are subjected to forces that exceed their impact tolerance. Injury to the facial bones might occur in one or more of the bones. Facial fractures most frequently occur in the zygoma or mandible and to a lesser extent in the maxilla, the incidence and etiology of mandibular fractures vary from one country to another because of social, cultural and environmental factors ${ }^{(1,2)}$.

Fracture morphology of maxillofacial trauma is often complex, so the clinicians should be familiar with the imaging findings. Various radiographic methods have been used for diagnosing maxillofacial trauma. Maxillofacial fractures require accurate radiologic diagnosis using multi-detector computed tomography (MDCT) and surgical management to prevent severe functional debilities and cosmetic deformity ${ }^{(3)}$.

Mandibular fractures are the next most common facial bone fractures after fractures of the nasal skeleton. These fractures occur most frequently as a result of the raised nature of this bone in the face. Mandibular fractures were classified into four types: median, paramedian, angle and condylar types. The most common mandibular fracture site was the condyle (33.6\%), followed by the angle $(21.7 \%)^{(1-4)}$.

The conventional preoperative planning with 2-D radiographs has not always provided sufficient information to understand the complex structures of the human body. It does not show anatomic details

* Lecturer of Oral and Maxillofacial Radiology, Faculty of Oral and Dental Medicine, Cairo University. 
in the cross sectional view, and the anatomical structures are shown overlapping. With the advances in radiology and computer technology in recent years, CT scan with 3-D reconstruction has enabled a better understanding of the morphological structures ${ }^{(5,6)}$.

The introduction of 3D tomography images for orthognathic planning and surgical simulation, associated with the rapidly emerging availability of this technology, has broadened the use and application of 3D imaging. Currently available three-dimensional (3D) software has been developed specifically to assist in the diagnosis, treatment planning, and prediction of outcomes related to orthognathic surgery to prevent preoperative and postoperative complications such as hemorrhage, permanent neural disorders, and unplanned fractures ${ }^{(7,8)}$.

$\mathrm{CT}$ is the imaging technique of choice to display the multiplicity of fragments, the rotation and dislocation degree, or any skull base involvement. Multidetector computed tomography (MDCT) allows high-quality multiplanar reformation (MPR) and isotropic viewing all of which improve the diagnostic power of this imaging modality, thus benefiting maxillofacial trauma patients and can detect the non-displaced fractures, also provide valuable three-dimensional (3D) morphology of the more complex injuries in maxillofacial trauma. In recent years, MDCT with MPR and 3D images has become a standard part of the assessment of facial injury because of the exquisite sensitivity of this imaging technique for fracture..$^{(3,9-11)}$.

\section{METHODOLOGY}

\section{Patients' selection:}

Ten adult patients (14 fractures) with suspected mandibular fracture were selected from the outpatient clinics of Oral and maxillofacial Surgery Department, Faculty of Oral and Dental Medicine, Cairo University. According to the following criteria:
1- Patient age. (30-50)

2- All were males.

Any diagnostic evaluation was postponed until the patient's condition had been stabilized by the oral surgeon.

\section{Patients' examination:}

All the patients were subjected to the following:

\section{Clinical Examination:}

- Thorough history of trauma.

- A thorough intra and extra-oral clinical examination was carried out in order to detect all the following signs and symptoms using inspection and palpation:

1- Facial asymmetry

2- Difficulty in opening and closing the mouth or trismus.

3- Extra or intraoral mucosal lacerations, edema, cut or open wounds and bleeding.

4- The presence and severity of Pain or tenderness upon palpation

5- Occlusal discrepancies.

6- Deviation of the mandible when trying to open the mouth.

7- Fractured or missed teeth.

4- Present no. of teeth and periodontal status.

\section{Radiographic Examination:}

\section{1-Multislice CT scan.}

The study was carried out in the Cairo-scan Radiology Center. Using multislice CT (MSCT, Somatom Sensation 16 slice machine (Siemens AG, Medical Solutions, Erlangen, Germany)) with Syngo system and VB10B software. 


\section{1- Patient Position:}

All the images were taken while the patient lies in supine position on the automatically moving scanning bed.

\section{2- Technical consideration:}

Scanning started from the level of the base of mandible to the glenoid fossa. Using $120 \mathrm{kVp}$, $80 \mathrm{~mA}$, slice thickness $0.75 \mathrm{~mm}$, Field of View (FOV) 140-200mm, Matrix Size 512x512 and Reconstruction Interval $0.5 \mathrm{~mm}$.

\section{3- Image processing:}

The archived data were originally stored on optical disk and then transferred to an advanced computer workstation (Siemens Navigator) to generate $3 \mathrm{D}$ reconstructed and $3 \mathrm{D}$ volumetric images allow for virtual visualization, manipulation and analysis of the area of trauma.

\section{4- Image analysis:}

- As the main cuts were in the axial plane, the software allows for 3D multiplanar reconstruction of the other two planes (coronal \& sagittal) which is used in assessment of mandibular fractures(Fig. 1,2,3,4).

** In an attempt to eliminate the intra-observer errors, the CT images were independently and separately assessed in a blind fashion at two different sessions with a minimum interval of 2 weeks between sessions to minimize personal memory errors.

** The obtained data and images were used to assess the mandibular fractures for the following: (The original data were transferred to a data worksheet summarizing the identified clinical and radiographic findings; these sheets were completed for all investigated cases)

1- Presence or absence of fracture.

2- Number and Location of fracture lines.

3- Degree and direction of displacement and step formation.
4- Presence or absence of bony fragments and Soft tissue involvement.

5- Areas of comminution.

\section{Radiographic Findings}

\section{- Computed Tomographic Findings}

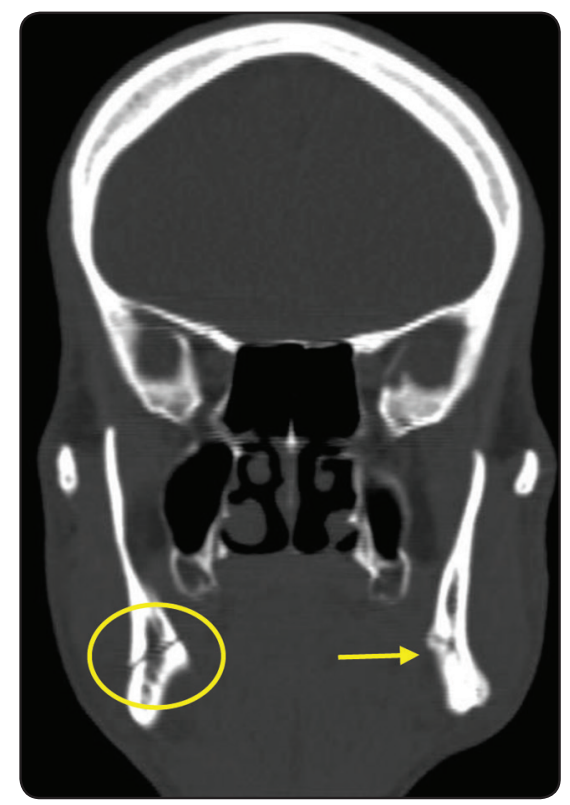

Fig. (1) Reformatted coronal image provides a better visualization of the complete oblique right body fracture clearly depicting the non displaced fracture line (circle) and incomplete oblique left body fracture (arrow).

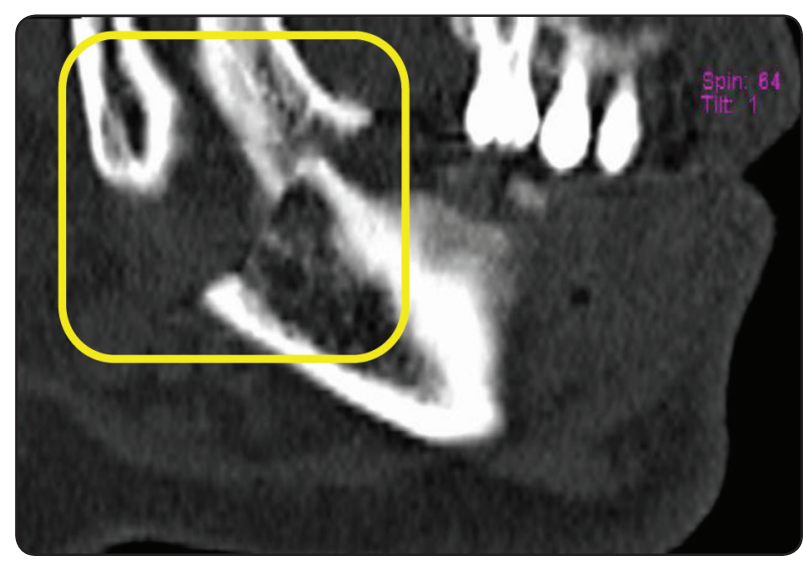

Fig. (2) The reformatted sagittal image perfectly depiciting the inferior and posterior displacement and separation of the right body fracture. There is a clear demonstration of the step formation with no overlap. 


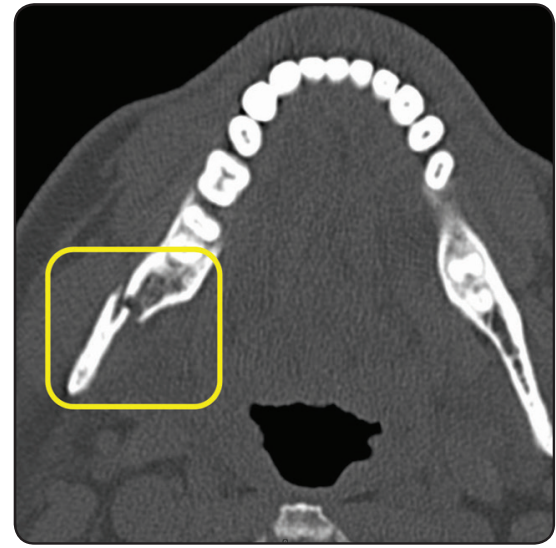

Fig. (3) The axial CT image showing the presence of one, complete oblique, laterally displaced right Angular fracture with a step formation and mild overlapping of bony segments on the right side.

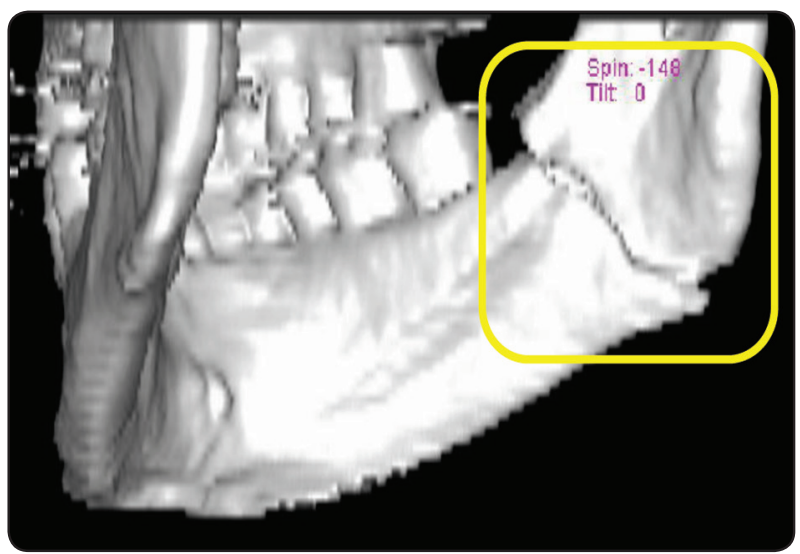

Fig. (4) The reconstructed 3D image showing the right side of the mandible and clearly localizing the oblique, inferiorly displaced angular fracture with step formation.

\section{STATISTICAL ANALYSIS}

** The data obtained was recorded, tabulated and statistically analyzed to reveal the validity, overall accuracy, and sensitivity of the applied imaging techniques (multislice CT and multiplannar reconstructed CT) in diagnosis of mandibular fractures.

** Qualitative data were presented as frequencies and percentages. Chi-square $\left(\mathrm{x}^{2}\right)$ test was used for studying the comparisons and associations between different variables.
** Sensitivity, Specificity, Positive, Negative predictive values and Diagnostic accuracy were calculated as follows:

$$
\begin{aligned}
& \text { - Sensitivity }(\%)=\frac{\text { True positive }}{\text { True positive }+ \text { False negative }} \times 100 \\
& - \text { Specificity }(\%)=\frac{\text { True negative }}{\text { False positive }+ \text { True negative }} \times 100
\end{aligned}
$$

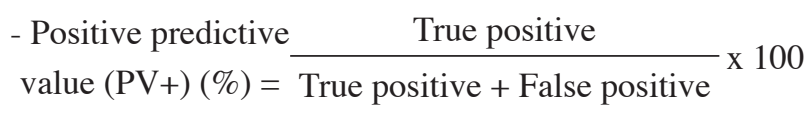

- Negative predictive
value $(\mathrm{PV}-)(\%)=$

$$
\text { - Diagnostic } \quad \frac{\text { True positive }+ \text { True negative }}{\text { Total number }} \times 100
$$

** An increase in sensitivity means a decrease in false negative cases, while an increase in specificity means a decrease in false positive cases.

** $\mathrm{PV}^{+}$is the probability that a person has the disease given that he tests positive, while $\mathrm{PV}^{-}$ is the probability that a person is disease-free given that he tests negative.

** The significance level was set at $\mathrm{P} \leq 0.05$. Statistical analysis was performed with SPSS 16.0 ${ }^{* \circledast}$ (Statistical Package for Scientific Studies) for Windows.

** The specificity could not be estimated because the diagnosis of true negative was not confirmed since surgical intervention occurred only for suspected fractures.

The axial and reformatted coronal images confirmed their superiority in revealing the exact number of fracture lines as they yielded the highest sensitivity and diagnostic accuracy values ( $100 \%$ and $100 \%$ for axial and $92.9 \%$ and $92.9 \%$ for the coronal).The least sensitive images were the sagittal

* ${ }^{\circledR}$ SPSS, Inc., Chicago, IL, USA. 
(76.6\% and $78.6 \%)$ and the reconstructed panoramic (78.6\% and $78.6 \%$ respectively).

Assessment of sensitivity and diagnostic accuracy of 2D-CT and 3D-CT techniques regarding detection of fractures revealed that all modalities were sensitive and highly accurate in detection of mandibular fractures where the axial and coronal images yielding the highest sensitivity and diagnostic accuracy (100\% and $100 \%$ respectively for both modalities). The reconstructed panoramic had the least sensitivity and accuracy $(85.7 \%$ and $85.7 \%$ ), while the sagittal and 3D images showed equivalent values $(92.9 \%$ and $92.9 \%$ respectively for each modality).

The axial images had lower sensitivity and diagnostic accuracy being similar to the sagittal and reconstructed panoramic in anatomic localization of the fracture lines $(78.6 \%$ and $78.6 \%$ respectively for the three modalities). On the other hand, the coronal and 3D images were very sensitive and accurate in localizing the investigated fractures.

Regarding displacement detection, the 3D showed superior sensitivity being comparable to the axial images in sensitivity (85.7\%) and showed higher diagnostic accuracy than the coronal images (78.6\% for coronal and $85.7 \%$ for 3D). Sagittal and reconstructed panoramic images had very poor sensitivity and diagnostic accuracy.

The reconstructed panoramic images and the 3D images yielded the least sensitivity $(71.4 \%$ and $78.6 \%$ respectively).Axial, coronal and 3D images showed the highest sensitivity in assessing facial asymmetry in comparison to the less sensitivity shown by the remaining imaging modalities.

\section{DISCUSSION}

Mandibular fracture is among the most common injury seen in the emergency room, for that reason oral and maxillofacial surgeons should have extensive knowledge of the mandibular anatomy, function and occlusion when treating any mandibular injury. Therefore, Effective clinical imaging must always be justified by positive influence on clinical management. In facial trauma, imaging provides information which is known to contribute to accurate diagnosis which enables the three-dimensional assessment of bony injuries, aids in decision-making process and improved clinical results ${ }^{(12-16)}$.

The introduction of multislice computed tomography (MSCT) represented a fundamental evolutionary step in the development and ongoing refinement of $\mathrm{CT}$ imaging techniques. A single MSCT scan can yield multiple, thin, overlapping slices that can be rapidly reconstructed, resulting in higher-quality reconstructed images and precluding the need for further patient radiation exposure. thereby decreasing the problem of patient movement during the data acquisition which has previously caused problems with image reconstruction and distortion in the final results that is why it was the technique of choice in the current study. ${ }^{(7)}$

Several 3D techniques have been developed to compensate for the drawbacks of 2D measurements. Early recognition of possible anatomical differences can prevent intraoperative and postoperative complications, such as sensorial, neurological, or skeletal disturbances and bleeding, which may compromise a patient's life. New software applications have been developed to enhance anatomical analysis pre-, intra, and postoperatively in a single software platform, to minimize surgical complications and improve diagnosis and treatment planning to obtain more predictable results after orthognathic surgery ${ }^{(8,17,18)}$.

According to Gaia et al 2011 ${ }^{(7)}$, both 2D-CT and 3D-CT techniques have similar sensitivity for the diagnosis of fractures in the mandibular region. However, they also reported that the 3D-CT image allowed a better visualization which is in agreement with the current study.

Both Costa e silva et al $\mathbf{2 0 0 3}^{\left({ }^{(19)}\right.}$ and Klenk and Kovacs $\mathbf{2 0 0 4}^{(\mathbf{2 0})}$ reported that $2 \mathrm{DCT}$ is 
essential for the surgeon to assess concomitant soft tissue complications and entrapment between the fragments but cannot replace the axial and coronal reconstructions in assessment of mandibular fractures.

Coronal CT is the best method to diagnose the extent of the fracture because the cut is taken perpendicular to the fracture plane. Certainly, this orientation does not exist in the axial CT plane of section because it is parallel and not perpendicular to the plane of the structure, rendering it less diagnostic in such cases. In consistent with these results are those reported by Klenk and Kovacs $\mathbf{2 0 0 4}^{(20)}$ who stated that coronal $2 \mathrm{DCT}$ is usually superior to axial 2DCT in identifying the exact anatomic position of fractures even in undisplaced or minimally displaced fractures.

According to Ogura et al $2014^{(3)}$ study, they consider that prevalence of condylar fractures using MDCT was higher than those of other reports because of the exquisite sensitivity of $\operatorname{MDCT}^{(\mathbf{9}, 10)}$.

Solar et al 2001 ${ }^{(21)}$ and Fatemitabar and Nikgoo $\mathbf{2 0 1 0}^{(22)}$ reported that $3 \mathrm{D}$ reconstructions of axial CT data were superior to 2D reformatting in furnishing the surgeon with information on the patient's individual anatomy.

Areas of comminutions were best revealed by the axial and reconstructed coronal in this study as they all showed high sensitivity, on the other hand, the reconstructed sagittal and reconstructed panoramic views showed a very low diagnostic accuracy regarding detection of comminution.

With respect to comminution of the condylar fracture, Costa e silva et al $\mathbf{2 0 0 3}^{(\mathbf{1 9 )}}$ proved that the 2D-CT examinations presented one false negative and one false positive result throughout their study period, while the 3D-CT presented none. Again these findings go against the current work.

Against the current study, is the work of Saigal et al $2005^{(23)}$ who stated that although 2D axial and coronal CT are more accurate and more sensitive than 3D reformatting, numerous studies have explored the utility of 3D imaging. Threedimensional images are created from the original 2D slices; therefore, no additional new information in the images could be gained, and artifacts may be produced in the reformation process.

The current results confirm previous reports that 3DCT imaging is useful for assessing the severity of facial injuries. I believe that this is probably because it provides the surgeons with graphic displays of the actual anatomic presentation that guides them in treatment planning and surgical approach in the operating room.

\section{CONCLUSION}

The present study strengthens the role of the 3D-CT technique as a complementary technique to the 2D-CT in the assessment of the mandibular fractures. 3DCT imaging has proved its validity as an exquisite tool in providing higher sensitivity in anatomic localization of mandibular fractures, in detecting comminution and bone displacement.

\section{REFERENCES}

1. Mohammadi $\mathrm{S}$ and Mohebbi $\mathrm{S}$ : Occurance of mandibulofacial injuries presenting to the otorhinolaryngology and head \& neck surgery department. The Journal of Craniofacial Surgery 2007; 18: 833-837.

2. Moliere: Beauty of face is a frail ornament, a passing flower, a momentary brightness belonging to the skin. Electronic Textbook 2008.

3. Ogura I, Sasaki Y and Kaneda T: Multidetector computed tomography of maxillofacial fractures. Japanese Dental Science Review 2014; 136: 1-5.

4. Lieger O, Zix J, Kruse A, Iizuka T: Dental injuries in association with facial fractures. J Oral MaxillofacSurg 2009; 67: 1680-4.

5. Albuquerque M. A. P, Gaia B. F and Cavalcanti M. G. P: Oral cleft volumetric assessment by $3 \mathrm{D}$ multislice computed tomographic images. Int. J. Oral Maxillofac. Surg 2011; 40: 1280-1288.

6. Li P, Tanga W, Liaoc C, Tana P, Zhanga J and Tian W: Clinical evaluation of computer-assisted surgical technique 
in thetreatment of comminuted mandibular fractures. Journal of Oral and Maxillofacial Surgery, Medicine, and Pathology 2014; 292: 1-5.

7. Gaia BF, Sales MAO, Perrella A, Fenyo-Pereira M, Cavalcanti MGP: Comparison between cone-beam and multislice computed tomography for identification of simulated bone lesions.Braz Oral Res 2011 Jul-Aug; 25(4):362-8.

8. Gaia B. F, Pinheiro L. R, Umetsubo O. S, Costa F. F, Cavalcanti M. G. P: Validity of three-dimensional computed tomography measurements for Le Fort I osteotomy. Int. J. Oral Maxillofac.Surg 2014; 43:197-203.

9. Sawazaki R, Lima Jr SM, Asprino L, Moreira RWF, de Moraes M: Incidence and patterns of mandibular condyle fractures. J OralMaxillofacSurg 2010; 68:1252-9.

10. Zhou HH, Liu Q, Cheng G, Li ZB:Aetiology, patternand treatment of mandibular condylar fractures in 549patients: a 22-year retrospective study. J CraniomaxillofacSurg 2013; 41: 34-41.

11. Ogura I, Sasaki Y, Kaneda T: Analysis of mandibular condylar andglenoid fossa fractures with computed tomography. EurRadiol 2014; 24: 902-6.

12. Reuben AD, Watt-Smith SR, Dobson D and Golding SJ: A comparative study of evaluation of radiographs, CT and 3D reformatted CT in facial trauma: what is the role of 3D?. The British Journal of Radiology 2005; 78: 198-201.

13. BriscoJ, Fuller K, Lee $\mathrm{N}$ and Andrew D:Cone beam computed tomography for imaging orbital trauma-image quality and radiation dose compared with conventional multislice computed tomography. British Journal of Oral and Maxillofacial Surgery 2014; 52: 76-80.

14. Goodday R. Management of fracture of the mandibular body and symphsis. Oral Maxillofacial SurgClin N Am 2013; 25: 601-616.

15. Frellesena C, Boettcher M, Wichmann J.L, Drieske Kerl J.M, Lehnert T, Nau C, Geiger E, Wutzler S, Ackermann H, Vogl T.J , Bauer R.W. Evaluation of a dual-room sliding gantry CT concept for workflow optimisation in polytrauma and regular in- and outpatient management. European Journal of Radiology 2015; 84: 117-122

16. Artigas Martína J.M., Martí de Graciab M, Claraco Vegac L.M, Parrilla Herranzd P. Radiology and imaging techniques in severe trauma.Med Intensiva. 2015; 39(1):49-59

17. Santos DT, Costa e Silva AP, Vanier MW, Cavalcanti MG: Validity of multislice computed tomography for diagnosis of maxillo-facial fractures using an independent workstation. Oral Surg Oral Med Oral Pathol Oral RadiolEndod 2004; 98: 715-20.

18. Popat H, Richmond S, Drage NA: New developments in: three-dimensional plan-ning for orthognathic surgery. J Orthod 2010; 37:62-71.

19. Costa e silva AP, Antunes JL and Cavalcanti MG: Interpretation of mandibular condyle fractures using 2Dand 3D-computed tomography.Brazillian Dental Journal 2003; 14(3): 203-208.

20. Klenk G and Kovacs A: Do we need three-dimensional computed tomography in maxillofacial surgery? Journal of Craniofacial Surgery 2004; 15:842-850.

21. Solar P, Gahleitner A, Bednar A, Rodinger S, Watzek G: Detection of the canal via shaded surface display and multiplanar reconstruction of CT data. J Oral Rehabil 2001; 28: 243-256.

22. Fatemitabar S.A and Nikgoo A: Multichannel Computed Tomography Versus Cone-Beam Computed Tomography: Linear Accuracy of In Vitro Measurements of the Maxilla for Implant Placement. Int $\mathbf{J}$ oral maxillofacial implants 2010; 25: 499-505.

23. Saigal K, Winokur RS, Finden S, Taub D and Pribitkin E: Useof three-dimensional computerized tomography reconstruction in complex facial trauma. Facial Plastic Surgery $2005 ; 21: 214-219$. 